\title{
Investigating the impact of SARS-CoV-2 infection on basic semen parameters and in vitro fertilization/intracytoplasmic sperm injection outcomes: a retrospective cohort study
}

Meng Wang ${ }^{1}$, Juan Hu${ }^{1}$, Bo Huang ${ }^{1}$, Qiyu Yang ${ }^{1}$, Sibo Liu ${ }^{2}$, Zhou Li ${ }^{1}$, Liu Yang ${ }^{1}$, Qingsong Xi3 ${ }^{3 \dagger}$, Lixia Zhu ${ }^{1 \dagger}$ and Lei $\operatorname{Jin}^{1 *+}$

\begin{abstract}
Background: This study aimed to evaluate the influences of SARS-CoV-2 infection on semen parameters and investigate the impact of the infection on in vitro fertilization (IVF) outcomes.

Methods: This retrospective study enrolled couples undergoing IVF cycles between May 2020 and February 2021 at Tongji Hospital, Wuhan. Baseline characteristics were matched using propensity score matching. Participants were categorized into an unexposed group (SARS-COV-2 negative) and exposed group (SARS-COV-2 positive) based on a history of SARS-CoV-2 infection, and the populations were 148 and 50 after matching, respectively. IVF data were compared between the matched cohorts. Moreover, semen parameters were compared before and after infection among the infected males. The main measures were semen parameters and IVF outcomes, including laboratory and clinical outcomes.

Results: Generally, the concentration and motility of sperm did not significantly differ before and after infection. Infected males seemed to have fewer sperm with normal morphology, while all values were above the limits. Notably, the blastocyst formation rate and available blastocyst rate in the exposed group were lower than those in the control group, despite similar mature oocytes rates, normal fertilization rates, cleavage rates, and high-quality embryo rates. Moreover, no significant differences were exhibited between the matched cohorts regarding the implantation rate, biochemical pregnancy rate, clinical pregnancy rate, or early miscarriage rate.

Conclusions: The results of this retrospective cohort study suggested that the semen quality and the chance of pregnancy in terms of IVF outcomes were comparable between the males with a history of SARS-CoV-2 infection and controls, although a decreased blastocyst formation rate and available blastocyst rate was observed in the exposed group, which needs to be reinforced by a multicenter long-term investigation with a larger sample size.
\end{abstract}

Keywords: SARS-CoV-2, COVID-19, Semen analysis, IVF outcome, Male fertility

*Correspondence: leijintjh@163.com

${ }^{\dagger}$ Qingsong Xi, Lixia Zhu and Lei Jin contributed equally.

1 Reproductive Medicine Center, Tongji Hospital, Tongji Medical College, Huazhong University of Science and Technology, 430030 Wuhan, China

Full list of author information is available at the end of the article

\section{Background}

The first coronavirus disease 2019 (COVID-19) case, caused by severe acute respiratory syndrome coronavirus 2 (SARS-CoV-2), was reported in December 2019 original author(s) and the source, provide a link to the Creative Commons licence, and indicate if changes were made. The images or other third party material in this article are included in the article's Creative Commons licence, unless indicated otherwise in a credit line to the material. If material is not included in the article's Creative Commons licence and your intended use is not permitted by statutory regulation or exceeds the permitted use, you will need to obtain permission directly from the copyright holder. To view a copy of this licence, visit http://creativecommons.org/licenses/by/4.0/. The Creative Commons Public Domain Dedication waiver (http://creativeco mmons.org/publicdomain/zero/1.0/) applies to the data made available in this article, unless otherwise stated in a credit line to the data. 
[1]. It was subsequently announced as a global pandemic in March 2020 by the World Health Organization (WHO). According to the WHO weekly situation report, this rapidly widespread disease had affected 220 countries and territories, with nearly 170 million confirmed cases and more than 3.5 million confirmed deaths by the end of May 2021 [2]. Worryingly, these numbers are still increasing, and the impacts of COVID-19 on public health care need persistent attention.

It is quite clear that the angiotensin-converting enzyme 2 (ACE2) receptors on cell surfaces is the main receptor for the entry of SARS-CoV-2 into cells [3], and theoretically, any tissue expressing ACE2 may be a potential target for SARS-CoV-2. However, accumulated studies have also demonstrated that, except in the lung, the expression levels of ACE2 were also significantly high in other organs, such as the kidney [4], intestines [5], and cardiovascular system [6, 7]. Notably, previous studies have shown that ACE2 also has high expression levels in spermatogonia, Sertoli cells, and Leydig cells in the male reproductive system [8], indicating that a history of SARS-CoV-2 infection may reduce male fertility function.

It has been previously demonstrated that males are more susceptible to acquire viral SARS-CoV-2 infections [9]. Changes in semen parameters, such as decreased motility and vitality, have been observed in infected males in some studies [10], while others have reported a comparable result in the infected patients and uninfected patients in terms of semen parameters [11]. The inconsistency of the conclusions may be attributed to the standards of sample selection of controls, which may act as selection bias of the results. Currently, there are no data comparing the semen parameters in individuals before and after infection within individuals, which would avoid the issues of both selection bias and confounding. Moreover, limited research has investigated the impacts of SARS-CoV-2 infection on gamete and embryonic development and implantation potential.

In this study, we collected assisted reproductive technology (ART) data from the largest in vitro fertilization (IVF) center in Wuhan, China, to evaluate the influences of infection on semen parameters and to investigate the impacts of a history of the infection in males on gamete and embryo development, as well as IVF outcomes.

\section{Materials and methods}

\section{Study design}

This retrospective cohort study enrolled all couples who underwent ART treatments from May 2020 to February 2021, at the Reproductive Medicine Centre, Tongji Hospital, Tongji Medical College, Huazhong University of Science and Technology. It was approved by the
Ethical Committee of Tongji Medical College ([2020] S066), and informed written consent for the ART procedures and possible data extraction were obtained from the patients. In the current study, each patient underwent routine serum SARS-CoV-2 antibody tests and PCR tests for detecting SARS-CoV-2 RNA at least three times, namely, at in the first visit to our center, before the controlled ovarian stimulation $(\mathrm{COH})$ procedure, and before oocyte retrieval. In addition to these two tests, digital chest radiographs were also performed for all patients to screen for any pulmonary lesions. Moreover, a detailed history taking was taken during the first visit, and the previous records of diagnosis and treatment for SARSCoV-2 infection were recorded in detail. According to the results of the abovementioned tests, the patients were divided into two groups: the "SARS-CoV-2 positive" group and the "SARS-CoV-2 negative" group. The inclusion criteria for the exposed group were as follows: males with (a) negative results for nucleic acid tests and (b) positive results for serum SARS-CoV-2 antibodies. Patients with the following cycle characteristics were excluded: (a) missing important information; (b) lost to follow-up; (c) oocyte donation cycles; (d) total or partial oocyte freezing cycles; and (e) females with a history of SARS-CoV-2 infection. Propensity score matching of 1:3 between the exposed group and the unexposed group was performed to create groups that were comparable for the matched characteristics.

\section{Semen analysis}

Freshly ejaculated semen samples were obtained by masturbation and ejaculation into sterile containers after an absence of sex for 2-7 days. After liquefaction for 30-60 $\mathrm{min}$ at room temperature, the samples were analyzed according to the published WHO criteria (fifth edition) [12]. Briefly, the lower reference limits of the semen parameters were as follows: $1.5 \mathrm{~mL}$ for the semen volume, $15 \mathrm{million} / \mathrm{mL}$ for the sperm concentration, 39 million for the total sperm number per ejaculate, $32 \%$ for progressive motility, $40 \%$ for total motility, and $4 \%$ for morphologically normal forms. A combination of manual Papanicolaou sperm staining and a computer-assisted sperm analysis (CASA) system was applied for semen analysis. The intra- or interobserver variability in semen assessment was adjusted by quality control of the CASA system each day and periodical personnel training.

\section{Sperm preparation for IVF}

Standard density-gradient centrifugation was performed for sperm selection as previously reported [13]. Briefly, up to $3 \mathrm{~mL}$ of semen was layered on pre-equilibrated 90\%/45\% gradient media (Vitrolife, Sweden) and centrifuged at $200 \mathrm{~g}$ for $20 \mathrm{~min}$. After washing with a sperm 
washing medium (Vitrolife, Sweden), the sperm pellet was resuspended in a $500 \mu \mathrm{L}$ medium for a swim-up for 30-60 min, and the top $300 \mu \mathrm{L}$ was collected for semen analysis and insemination. If the concentrations of optimized sperm were above $5 \times 10^{6} / \mathrm{mL}$, regular IVF was performed; otherwise, intracytoplasmic sperm injection (ICSI) was chosen.

\section{Oocyte retrieval and embryo culture}

Controlled ovarian stimulation $(\mathrm{COH})$ was performed based on previous studies [14]. The $\mathrm{COH}$ protocols were mainly the gonadotropin-releasing hormone $(\mathrm{GnRH})$ agonist protocol, the $\mathrm{GnRH}$ antagonist protocol, and other protocols, such as mild stimulation and luteal phase stimulation protocols. Recombinant human chorionic gonadotrophin (HCG) was intramuscularly administered when there were two to three dominant follicles with a diameter over $18 \mathrm{~mm}$. Oocytes were retrieved $36-38 \mathrm{~h}$ after the HCG trigger.

The presentation of two pronuclei (2PN) $16-18 \mathrm{~h}$ after insemination was regarded as normal fertilization. Embryos were cultured to the cleavage stage in G1-plus medium (Vitrolife, Gothenburg, Sweden) until Day 3. One or two embryos with high quality were freshly transferred; the surplus embryos were cryopreserved on Day 3 or the culture was extended in G2-plus medium (Vitrolife, Gothenburg, Sweden) up to Day 5 or 6 until the embryos reached the blastocyst stage, and available blastocysts were cryopreserved. The morphological scoring systems of cleavage-stage embryos and blastocysts were described previously in detail $[15,16]$. Additionally, highquality embryos at the cleavage stage were clearly defined in previous studies [15]. On Day 5 or 6, blastocysts with a grade of $3 \mathrm{BC}$ or higher were considered to be available for cryopreservation.

\section{Serum SARS-CoV-2 antibody tests and nucleic acid tests}

According to the manufacturer's instructions, serum SARS-CoV-2 antibodies, including IgG (sensitivity 98.5\%, specificity $100 \%$ ) and IgM (sensitivity $84.3 \%$, specificity 98.5\%), were detected using chemiluminescent immunoassays (C86095G, C86095M, YHLO Biotech, Shenzhen, China) [17]. The cutoff values for IgG and IgM were both 10 , and a serum antibody value no less than $10 \mathrm{AU} / \mathrm{mL}$ was considered to be positive.

Real-time polymerase chain reaction (RT-PCR, 20,203,400,749, DA AN GENE, Guangzhou, China) was utilized to detect the presence of SARS-CoV-2 in the viral RNA of the specimens collected from nasopharyngeal swabs. The open reading frame $1 \mathrm{ab}$ (ORF1ab) and $\mathrm{N}$ genes of SARS-CoV-2 were the target genes for RT-PCR, and the primers were described previously [18]. The analytical sensitivity for RT-PCR was 500 copies $/ \mathrm{mL}$, and positive results for both genes were equipped with $\mathrm{Ct}$ values less than 30 .

\section{Assessment of IVF outcomes}

The laboratory outcomes were the developmental parameters of the embryos at different stages, including the mature oocyte rate, damaged oocyte rate, normal fertilization rate, abnormal fertilization rate, cleavage rate, high-quality embryo rate, blastocyst formation rate, and available blastocyst rate. Moreover, the clinical outcomes were mainly the implantation rate, biochemical pregnancy rate, clinical pregnancy rate, and early miscarriage rate. The details of the computing methods have been previously described [14, 19] except for several slight modifications. The blastocyst formation rate was defined as the number of blastocysts formed divided by the number of Day 3 embryos for extended culture. Similarly, the available blastocyst rate was the number of blastocysts for cryopreservation divided by the number of Day 3 embryos for extended culture. A clinical pregnancy was defined as an active fetal heart rate in the uterus detected using ultrasound five weeks after embryo transfer, whereas a biochemical pregnancy was regarded as a positive result of an HCG measurement. The denominator of the clinical pregnancy rate and biochemical pregnancy rate was the number of embryo transfer cycles. An early miscarriage referred to the loss of a fetal heart rate within the first three months.

\section{Statistical analyses}

The Statistical Package for Social Sciences software (version 26.0; SPSS, IBM, USA) was used for data analyses. Kolmogorov-Smirnov or Shapiro-Wilk normality tests were utilized for the normality tests of continuous variables. Nonnormally distributed variables are presented as medians (first quartile, third quartile), while the categorical variables are presented as the $\%(\mathrm{n})$. Differences between the groups were analyzed using the nonparametric rank-sum test (Mann-Whitney $U$ test) for continuous variables and the chi-squared test or Fisher's exact test for categorical variables as appropriate. The Wilcoxon rank test was used to evaluate alterations in semen parameters before and after infection in the same individuals.

Propensity scores were calculated using logistic regression based on the following characteristics: male age (year); female age (year); female body mass index (BMI, $\mathrm{kg} / \mathrm{m}^{2}$ ); basal follicle stimulation hormone (FSH, $\mathrm{mIU} /$ $\mathrm{mL}$ ); basal anti-Müllerian hormone (AMH, $\mathrm{ng} / \mathrm{mL})$; basal antral follicle counting (AFC); infertility type (primary or secondary); infertility duration (years); infertility causes (female factor, male factor, and both female and 


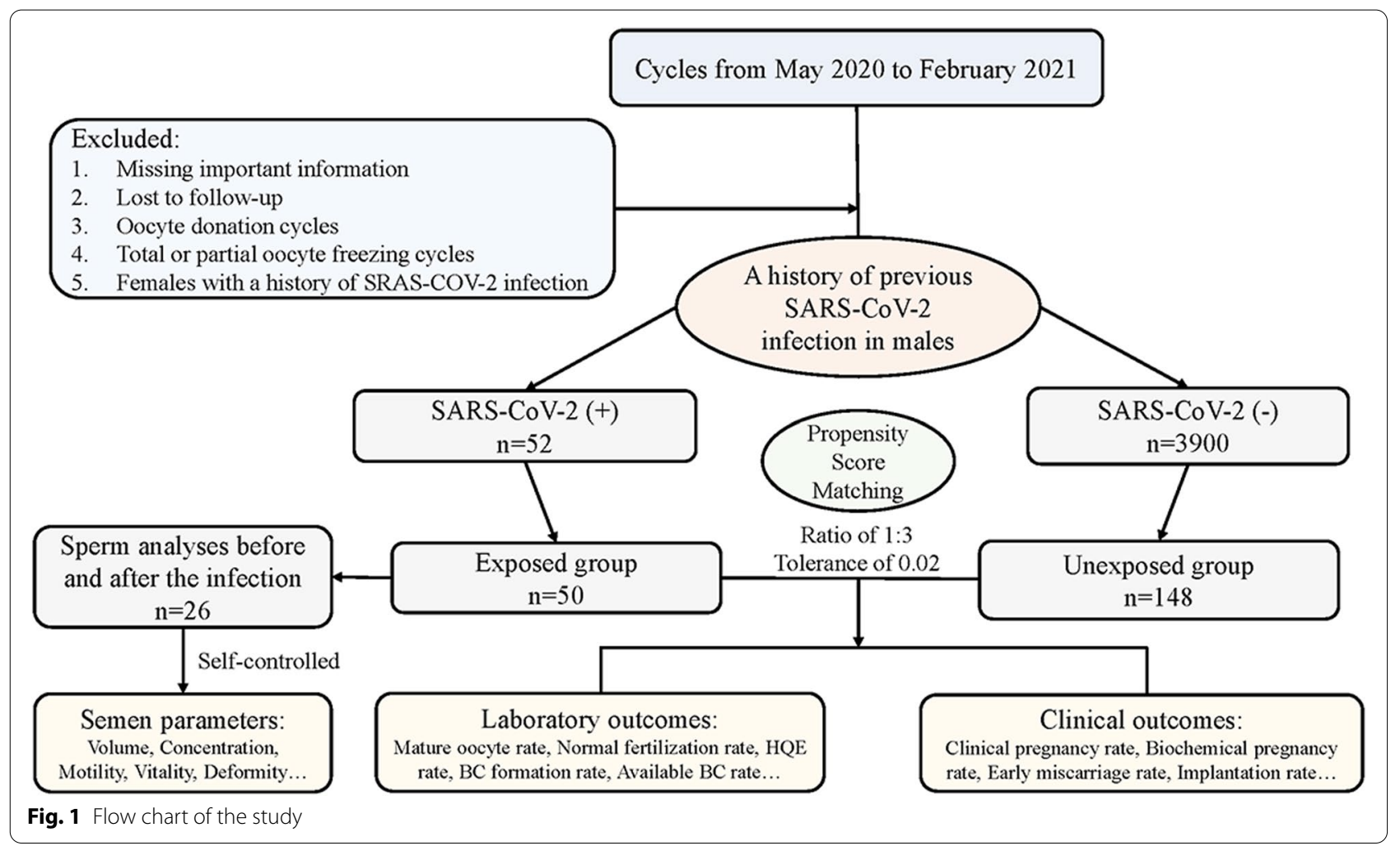

male factor); number of ART sessions; operation types (IVF and ICSI); $\mathrm{COH}$ protocols (GnRH agonist protocol, GnRH antagonist protocol, and other protocols); gonadotrophin duration (days); gonadotrophin dosage (IU); estradiol on the day of HCG administration $(\mathrm{pg} / \mathrm{mL})$; progesterone on the day of HCG administration $(\mathrm{ng} / \mathrm{mL})$; and endometrium thickness on the day of HCG administration $(\mathrm{mm})$. These characteristics were chosen to create cohorts that should be similar aside from their exposure to SARS-CoV-2 infection. Matching was performed using the nearest neighbor random matching algorithm with a 1:3 ratio and a 0.02 tolerance without replacement. Two-tailed $P$ values $<0.05$ were considered statistically significant.

\section{Results}

According to the results of the SARS-CoV-2 antibody tests, the individuals were divided into $\operatorname{IgG} / \operatorname{IgM}$ - $(n=3900)$ and IgG/IgM + $(n=52)$ groups. Then, propensity score matching was performed: 148 patients were included in the unexposed group, and 50 were included in the exposed group after matching (Fig. 1). No significant differences were observed between the groups after matching in terms of the basal characteristics (Table 1). The distribution of propensity scores and standard deviations before and after matching are presented in Fig. 2. The overlap in densities represented the balance of the distribution between the compared cohorts, and it was obvious that the patients were well matched after propensity score matching.

After matching, 50 male COVID-19 patients were included, and Table 2 shows the COVID-19-related demographic and clinical characteristics of the males infected with SARS-CoV-2. Among these patients, 6 $(6 / 50,12.0 \%)$ described mild symptoms, mainly fever, cough, headache, muscle pain, and fatigue, yet they did not have any radiological changes in the lungs. One patient also reported anosmia. Seven participants (7/50, $14.0 \%)$ were classified as having moderate infection with typical chest CT manifestations. The remaining 37 (37/50, 74.0\%) patients had asymptomatic infections. These patients were diagnosed between January and March 2020. At least four months had passed from the first diagnosis of the SARS-CoV-2 infection to IVF treatment.

Among the 50 males with a history of SARS-CoV-2 infection, 26 have had undergone one previous semen analysis before infection. The semen parameters of the males before and after SARS-CoV-2 infection are summarized in Table 3. Generally, there were no significant 
Table 1 Baseline characteristics after matching

\begin{tabular}{|c|c|c|c|}
\hline & Unexposed $(n=148)$ & Exposed $(n=50)$ & $P$ value \\
\hline Male age, yr & $33(30,39)$ & $33(31,37)$ & 0.946 \\
\hline Female age, yr & $32(29,37)$ & $32(30,35)$ & 0.578 \\
\hline Female BMI, kg/m² & $21.1(21.9,22.4)$ & $21.6(19.8,23.8)$ & 0.536 \\
\hline $\mathrm{FSH}, \mathrm{mIU} / \mathrm{mL}$ & $7.50(6.20,9.10)$ & $7.1(6.4,8.9)$ & 0.633 \\
\hline $\mathrm{AMH}, \mathrm{ng} / \mathrm{mL}$ & $2.28(1.03,4.47)$ & $2.09(1.36,3.52)$ & 0.855 \\
\hline AFC & $9(5,17)$ & $8(5,15)$ & 0.896 \\
\hline Infertility type & & & 0.688 \\
\hline Primary, \% & $62.8(93)$ & $66.0(33)$ & \\
\hline Secondary, \% & $37.2(55)$ & $34.0(17)$ & \\
\hline Infertility duration, yr & $2(1,3)$ & $1(1,3)$ & 0.512 \\
\hline Infertility causes & & & 0.921 \\
\hline Female factors, $\%$ & $68.9(102)$ & $70.0(35)$ & \\
\hline Male factors, $\%$ & $14.2(21)$ & $13.6(6)$ & \\
\hline Both female and male factors, $\%$ & $16.9(25)$ & $17.2(9)$ & \\
\hline No. of ART sessions & $1(1,2)$ & $1(1,2)$ & 0.513 \\
\hline Operation types & & & 0.738 \\
\hline$I V F, \%$ & $54.7(81)$ & $52.0(26)$ & \\
\hline $\mid \mathrm{CSI}, \%$ & $45.3(67)$ & $48.0(24)$ & \\
\hline $\mathrm{COH}$ protocol & & & 0.976 \\
\hline GnRH-agonist, \% & $32.4(48)$ & $34.0(17)$ & \\
\hline GnRH-antagonist, \% & $48.6(72)$ & $48.0(24)$ & \\
\hline Others $^{a}, \%$ & $18.9(28)$ & $18.0(9)$ & \\
\hline Gn duration, d & $10(9,11)$ & $10(8,11)$ & 0.664 \\
\hline Gn dosage, IU & $2400(1913,3000)$ & $2550(1892,3094)$ & 0.634 \\
\hline Estradiol on HCG day, pg/mL & $1827(1050,2680)$ & $1709(1077,2608)$ & 0.925 \\
\hline Progesterone on HCG day, ng/mL & $0.71(0.43,1.05)$ & $0.72(0.32,1.07)$ & 0.924 \\
\hline Endometrium thickness on HCG day, mm & $10.5(8.7,13.0)$ & $10.8(8.9,12.1)$ & 0.805 \\
\hline
\end{tabular}

\section{Note:}

BMI, body mass index; FSH, follicle stimulation hormone; $\mathrm{AMH}$, anti-müllerian hormone; AFC, antral follicle counting; IVF, in vitro fertilization; ICSI, intracytoplasmic sperm injection; $\mathrm{COH}$, controlled ovarian hyperstimulation; $\mathrm{GnRH}$, gonadotrophin releasing hormone; Gn, gonadotropin; HCG, human chorionic gonadotropin

a Others: including mild stimulation and luteal phase stimulation protocols

Continuous variables were presented as median (first quartile, third quartile)

Categorical variables were presented as $\%$ (n)

$P<0.05$ was considered statistically significant

differences before and after infection, except for the percentage $(P<0.001)$ and number $(P=0.030)$ of sperm with morphologically normal forms. After infection, males seemed to have fewer normal sperm in terms of sperm morphology, although all the values were still within the normal reference range.

The data on embryo laboratory outcomes are presented in Table 4. The proportions of mature oocytes, damaged oocytes, normally fertilized oocytes, abnormally fertilized oocytes, normally cleaved embryos, and high-quality embryos at Day 3 were comparable between the groups. Notably, the blastocyst formation rate $(P<0.001)$ and available blastocyst rate $(P=0.005)$ in the exposed group were dramatically lower than those in the control group, indicating possibly impaired developmental potentials from the cleavage stage to the blastocyst stage of embryos from infected males.

Twenty-six (52.0\%) cycles in the exposed group and $72(48.7 \%)$ in the control group after matching (Table 5) were fresh embryo transfer cycles on Day 3. For each cycle, 1 or 2 embryos were transferred as appropriate. The clinical outcomes of the embryos for both groups were similar, and no statistically significant differences were exhibited between the matched cohorts regarding the implantation rate, biochemical pregnancy rate, clinical pregnancy rate, and early miscarriage rate. 


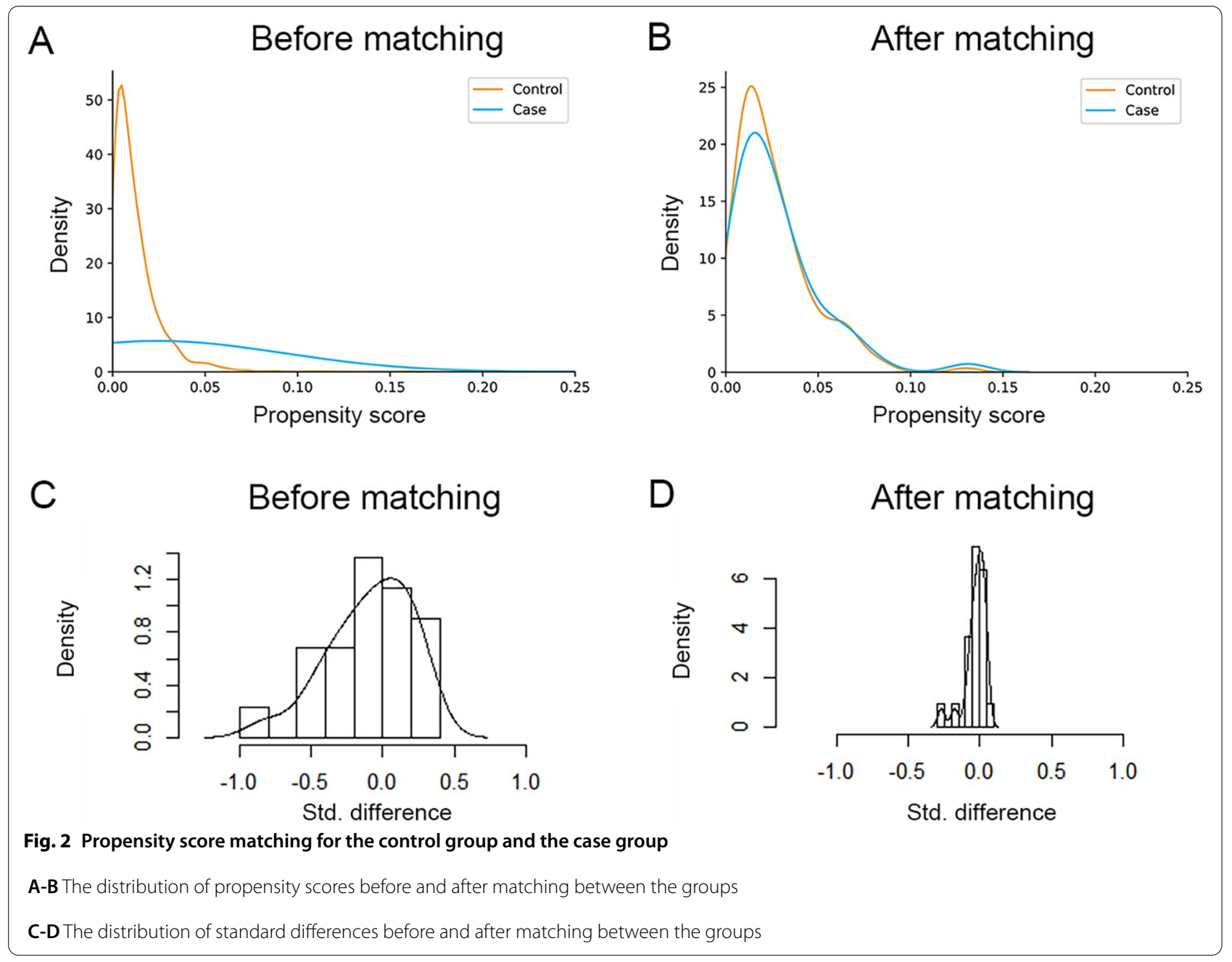

Table 2 Clinical characteristics of males infected with SARSCoV-2 $(n=50)$

\begin{tabular}{|c|c|}
\hline Clinical characteristic & Data \\
\hline Age, yr & $33(31,37)$ \\
\hline Hospitalization & $6 / 50$ \\
\hline $\begin{array}{l}\text { Time between the semen collections before and } \\
\text { after infection, } \mathrm{m}^{\text {a }}\end{array}$ & $15.50(11.75,24.00)$ \\
\hline \multicolumn{2}{|l|}{ SARS-CoV-2 antibodies positive ${ }^{b}$} \\
\hline IgM positive & $2 / 50$ \\
\hline IgG positive & $50 / 50$ \\
\hline Oropharyngeal swab positive ${ }^{b}$ & $0 / 50$ \\
\hline \multicolumn{2}{|l|}{ Severity of the infection } \\
\hline Asymptomatic & $37 / 50$ \\
\hline Mild & $6 / 50$ \\
\hline Moderate & $7 / 50$ \\
\hline
\end{tabular}

Note:

Continuous data presented as median (first quartile, third quartile)

${ }^{a}$ Twenty-six of these patients have experienced semen analyses before and after the infection

${ }^{\mathrm{b}}$ Results within one week before oocytes retrieval

\section{Discussion}

The current study enrolled male patients with a history of SARS-CoV-2 infection, analyzed the semen parameters of individuals before and after infection, and compared the IVF outcomes between infected and uninfected males. The percentage and number of sperm with normal morphology decreased compared with the pre-disease period, although all the parameters were still in the normal ranges. The IVF data analyses demonstrated that SARS-CoV-2 infection in males may not greatly impair IVF outcomes in terms of clinical outcomes, while a decreased blastocyst formation rate and available blastocyst rate was observed in the infected group, revealing a potential negative impact on embryonic development competency.

SARS-CoV-2 entering host cells through ACE2 receptors is a well-known fact [20], and the organs of the male reproductive system, including the testes, are reported to highly express ACE2 [21], making sperm susceptible to infection. Some studies investigated semen samples from 
Table 3 Semen parameters of males before and after matching $(n=26)$

\begin{tabular}{|c|c|c|c|c|}
\hline Semen parameters & Before & After & D-value ${ }^{a}$ & $P$ value \\
\hline Volume, $\mathrm{mL}$ & $3.0(2.0,3.4)$ & $2.7(2.3,3.6)$ & -0.3 & 0.247 \\
\hline Semen concentration, $10^{6} / \mathrm{mL}$ & $67.6(31.7,125.2)$ & $62.5(29.8,95.3)$ & 5.3 & 0.118 \\
\hline Total no. of sperm per ejaculate, $10^{6}$ & $198.1(99.4,343.7)$ & $203.4(108.4,251.1)$ & 15.4 & 0.304 \\
\hline Progressive motility, $\%$ & $48.1(21.1,67.6)$ & $43.0(27.3,61.8)$ & 3.0 & 0.551 \\
\hline Total no. of progressive motility, $10^{6}$ & $72.0(30.3,169.8)$ & $61.5(32.7,151.5)$ & 9.9 & 0.269 \\
\hline Complete motility, \% & $50.7(32.5,70.2)$ & $45.5(29.8,64.8)$ & 3.6 & 0.228 \\
\hline Total no. of complete motility, $10^{6}$ & $87.1(32.6,199.4)$ & $64.1(34.3,158.9)$ & 9.1 & 0.191 \\
\hline Immotile, \% & $49.3(29.9,67.7)$ & $52.5(32.5,63.3)$ & -2.0 & 0.493 \\
\hline Total no. of immotile, $10^{6}$ & $65.6(43.4,141.5)$ & $76.5(58.7,125.5)$ & -2.9 & 0.929 \\
\hline Normal forms, \% & $6.0(5.0,9.3)$ & $4.0(4.0,5.0)$ & 2.0 & $<0.001$ \\
\hline Total no. of normal forms, $10^{6}$ & $10.9(4.7,28.5)$ & $8.5(4.0,12.5)$ & 2.0 & 0.030 \\
\hline
\end{tabular}

Note:

Data were presented as median (first quartile, third quartile)

$P<0.05$ was considered statistically significant

a $\mathrm{D}$-value referred to the difference of medians before and after infection

Table 4 Embryo Laboratory outcomes after matching

\begin{tabular}{|c|c|c|c|c|}
\hline & Unexposed $(n=148)$ & Exposed $(n=50)$ & $P$ value & OR $95 \% \mathrm{Cl}$ \\
\hline No. of oocytes retrieved & 1620 & 579 & & \\
\hline Mature oocyte rate, $\%$ & $83.1(1347)$ & $85.0(492)$ & 0.308 & $0.87(0.67,1.13)$ \\
\hline Damaged oocyte rate, $\%$ & $4.0(64)$ & $3.1(18)$ & 0.359 & $1.28(0.75,2.17)$ \\
\hline Normal fertilization rate, $\%$ & $71.3(960)$ & $69.7(343)$ & 0.516 & $1.08(0.86,1.35)$ \\
\hline Abnormal fertilization rate, $\%$ & $8.4(113)$ & $10.4(51)$ & 0.118 & $0.79(0.56,1.12)$ \\
\hline Cleavage rate, $\%$ & $97.0(931)$ & $98.8(339)$ & 0.061 & $0.38(0.13,1.09)$ \\
\hline High quality embryo rate, $\%$ & $50.2(467)$ & $49.6(168)$ & 0.849 & $1.02(0.80,1.31)$ \\
\hline Blastocyst formation rate ${ }^{a}, \%$ & $72.9(554)$ & $57.1(165)$ & $<0.001$ & $2.02(1.52,2.68)$ \\
\hline Available blastocyst rate ${ }^{\mathrm{b}}, \%$ & $51.8(394)$ & $42.2(122)$ & 0.005 & $1.47(1.12,1.94)$ \\
\hline
\end{tabular}

Note:

OR, Odds ratio; $\mathrm{Cl}$, confidence interval

Categorical variable was presented as $\%(n)$

$P<0.05$ was considered statistically significant

a Blastocyst formation rate = the number of blastocyst formation/ the number of day 3 embryos for extended culture

b Available blastocyst rate $=$ the number of blastocyst for cryopreservation/ the number of day 3 embryos for extended culture

recovered and acutely infected SARS-CoV-2 patients, while no RNA of the virus was detected by RT-PCR [22-25]. Several studies solely focused on the investigation of SARS-CoV-2 in semen from patients in the acute phase of the infection [26-28]. Most of the studies showed no detection of the virus in the semen, although one reported the presence of SARS-CoV-2 in 6 out of 38 males [28]. However, whether the viruses exist in the semen of males with a history of SARS-CoV-2 infection remains underexplored. Moreover, it is still unclear whether SARS-CoV-2 indirectly affects male reproductive function by means of immune interference or other ways.
Semen parameters are most frequently used to assess male fertility. The exact impact of SARS-CoV-2 infection on semen parameters in infected males is still a matter of debate. In our study, 26 out of 50 patients had undergone one previous semen analysis before infection, and the comparison of semen parameters before and after infection indicated that semen quality was not greatly impaired by the infection. These results were consistent with those previously reported [29], in which COVID19 had no specific negative effect on male reproductive function. However, several studies reported impairments in semen, such as azoospermia [30] and oligozoospermia [31], after recovery from the disease. An observational 
Table 5 Clinical outcomes after matching

\begin{tabular}{|c|c|c|c|c|}
\hline & Unexposed $(n=148)$ & Expose $(n=50)$ & $P$ value & OR $95 \% \mathrm{Cl}$ \\
\hline Fresh embryo transfer cycles & 72 & 26 & & \\
\hline No. of embryos transferred & 84 & 28 & 0.342 & \\
\hline $1, \%$ & $83.3(60)$ & $92.3(24)$ & & \\
\hline $2, \%$ & $16.7(12)$ & $7.7(2)$ & & \\
\hline Implantation rate, $\%$ & $29.8(25)$ & $39.3(11)$ & 0.350 & $0.63(0.26,1.54)$ \\
\hline Biochemical pregnancy rate, $\%$ & $47.2(34)$ & $46.2(12)$ & 0.925 & $1.04(0.42,2.57)$ \\
\hline Clinical pregnancy rate, $\%$ & $33.3(24)$ & $42.3(11)$ & 0.413 & $0.68(0.27,1.71)$ \\
\hline Early miscarriage rate, $\%$ & $25.0(6)$ & $0.0(0)$ & 0.146 & N/A \\
\hline
\end{tabular}

Note:

OR, Odds ratio; $\mathrm{Cl}$, confidence interval; N/A, not applicable

Categorical variable was presented as $\%(n)$

$P<0.05$ was considered statistically significant

study also showed an increased proportion of apoptotic cells in the testicles and epididymis of patients who died from COVID-19 [31]. The severity and phases of the disease and the viral load of the blood may be one of the possible reasons, as well as the confounding bias caused by the selection of controls $[11,32]$. Nevertheless, the current study was a self-controlled study to eliminate the influence of individual differences; meanwhile, propensity score matching was performed to eliminate the imbalance of the number and distribution of participants between groups. Thus, our findings are much more convincing and reliable. In addition, a multicenter study with a population of 69 suggested decreased motility and vitality in mildly infected patients and reduced semen parameters in moderately infected patients before and after COVID-19, revealing a deteriorative impact of COVID19 on semen parameters over a short time period [10]. The semen analyses in our study were performed at least four months after the viral infection, and the participants had already experienced a long period of recovery for reproductive function. The former multicenter study only investigated the short-term effects, which may be the main reason for the inconsistency between the results. The study also suggested that male reproductive function might be impaired and decrease over a short period of time, while in the long run, it might recover and return to normal.

It is noteworthy that although the percentage and number of sperm with morphologically normal forms were lower after infection, the parameter was still in the normal range. Similarly, statistically significant differences within the normal range were also observed in other studies $[22,26]$. One of the studies included 74 recovered male patients and investigated testicular function. No virus was detected in the semen samples, and the levels of reproductive hormones and semen parameters remained within the normal limits, despite a lower total count and total motility in the semen samples of the recovered patients. In another study, 18 semen samples from the recovered men were tested, and the the absence of the virus in the semen and relatively normal results of semen analyses in individuals with and without fever during infection were also presented. Person-to-person spread, mainly via close contact and respiratory droplets, has been proven to be the primary COVID-19 transmission route [33]. Previous studies have demonstrated that the semen viral load is related to the blood viral load, and only when there was a higher blood viral load and viremia caused by the entry of viruses from the respiratory tract to the peripheral blood, is the male reproductive system afftected [34]. Moreover, the blood-testis barrier is a crucial defender against pathogen invasion from the peripheral blood to the testes [35]. Therefore, as presented in previous studies and our study, SARS-CoV-2 was absent in semen, and the infection in males may not greatly impair male reproductive function, and the semen parameters were still in the normal range.

Interestingly, in the current study, compared to those in the control group, the blastocyst formation rate and available blastocyst rate were much lower in the exposed group, which drew our attention. The developmental window from the cleavage stage to the blastocyst stage was vulnerable to interference, and some other viruses, such as the Zika and mumps viruses [36], were reported to impair early embryonic development. Another study also emphasized the potential risks of SARS-CoV-2 infection on gamete and embryo development [37]. Moreover, a lower blastocyst formation rate was unexpectedly observed in females with a history of mild or asymptomatic SARS-CoV-2 infection in our previous study [38], indicating that the impacts of the infection on embryonic development, especially subsequent fetal growth 
and development, are worthy of attention. It was shown that a previous infection in males did not impact clinical outcomes in terms of the implantation and pregnancy rate in fresh cycles. However, due to the decreased blastocyst formation rate and available blastocyst rate in the exposed group, it was unclear whether a previous SARS-CoV-2 infection impaired the chance of a clinical pregnancy in subsequent frozen-thawed embryo transfer (FET) cycles and the cumulative pregnancy chance in multiple cycles because of the decreased number of harvested embryos. Moreover, the impaired parameters of blastocysts may interfere with the maternal complications and neonatal outcomes. Thus, a long-term followup is needed.

Successful embryo development is affected and controlled by numerous intrinsic and extrinsic factors. Gamete quality, the culture environment, and many other factors may also have consequences on embryo developmental potential [39]. Epigenetic modifications, such as DNA methylation and histone modifications, play a crucial role in the initiation of embryonic transcription and embryonic lineage differentiation with the activation of the embryonic genome by Day 3 after fertilization [40]. The dynamics of epigenetic changes make embryos susceptible to perturbation, which may subsequently impact downstream embryonic development [41]. There are several parameters for evaluations of male fertility, and semen parameter analysis is the most common and frequently used method. The activation of the male genome is initiated after the process of fertilization and occurs around the blastocyst stage [42], making IVF outcomes a more precise and better indicator to assess sperm competence and viability. Previous studies have also detected the transcriptional expression of hominid-specific retrotransposons, the dysregulation of which could induce diseases, and have shown that these genes had a higher transcriptional level and better chromatin accessibility in early human embryos [43, 44]f. Our findings revealed decreased blastocyst development competency in infected patients. Whether SARS-CoV-2 infection in males negatively impacts embryonic epigenetics, resulting in health disorders of neonates and offspring, needs further observation, and close follow-up of the neonates is also necessary and recommended.

To the best of our knowledge, the current study is the first to enroll male patients with a history of SARS-CoV-2 infection to compare IVF outcomes between infected and uninfected males. However, several limitations existed in this study. First, it was a single-center retrospective cohort study with a limited sample size. More data from multiple centers worldwide are needed to overcome the boundedness of the sample size and region. Second, some patients had only undergone one semen analysis before and after the infection, while the dynamic nature of the sperm may make the comparisons of our results inaccurate. Moreover, only the patients in the exposed group were included, and there was a lack of a control arm. In addition, only semen analysis was used for evaluating male fertility, and more data on other evaluation indices are needed for comparison. The lack of data on live birth and cumulative pregnancy outcomes can be considered another limitation, and long-term close follow-up is needed. Furthermore, the majority of our participants had only with mild clinical symptoms or asymptomatic infections, which may not reflect the exact impact of SARS-CoV-2 on IVF outcomes for infected males.

In conclusion, the results of this retrospective cohort study suggested that semen quality and pregnancy chance in terms of IVF outcomes were comparable between males with a history of SARS-CoV-2 infection and controls, although a decreased blastocyst formation rate and available blastocyst rate were observed in the exposed group, which needs to be reinforced by a multicenter long-term investigation with a larger sample size.

\section{Abbreviations \\ ACE2: angiotensin-convertingenzyme 2; AFC: antral follicle counting; $\mathrm{AMH}$ anti-müllerian hormone; ART: assisted reproductivetechnology; BMI: body mass index; $\mathrm{COH}$ : controlled ovarianstimulation; COVID-19: coronavirus disease 2019; FSH: follicle stimulationhormone; GnRH: gonadotropin-releas- inghormone; HCG: human chorionic gonadotrophin; ICSI: intracytoplasmic sperminjection; IVF: in vitro fertilization; PN: pronuclei; SARS-CoV-2: severe acute respiratorysyndrome coronavirus 2 .}

\section{Acknowledgements}

We would like to express heartfelt gratitude to our colleagues in Reproductive Medicine Center, Tongji Hospital, Tongji Medical College, Huazhong University of Science and Technology.

\section{Authors' contributions}

$Q X, L Z$, and $L J$ conceived the study and have full access to the raw data; MW and LZ wrote the paper; ZL, BH, LY and JH collected the data; MW, QY, and SL analyzed the data. All authors read and approved the final manuscript.

\section{Funding}

This study was supported by the Health Commission of Hubei Province Scientific Research Project (WJ2021M110, receipted by LZ), the Fundamental Research Funds for the Central Universities (2021yjsCXCY095, receipted by $\mathrm{MW}$ ), and the National Key Research and Development Project (2018YFC1002103, receipted by LJ).

\section{Availability of supporting data}

The data that supportthe findings of this study are available from the corresponding author uponreasonable request

\section{Declarations}

Ethics Approval and Consent to participate

This retrospective study was approved by the Ethical Committee of Tongji Medical College ([2020]S066) ) without the need for informed consent.

Consent for publication

Not applicable. 


\section{Competing of interests}

All authors have no conflicts of interest to declare.

\section{Author details}

${ }^{1}$ Reproductive Medicine Center, Tongji Hospital, Tongji Medical College, Huazhong University of Science and Technology, 430030 Wuhan, China. ${ }^{2}$ Department of Nutrition and Food Hygiene, Hubei Key Laboratory of Food Nutrition and Safety, Tongji Medical College, Huazhong University of Science and Technology, Wuhan, China. ${ }^{3}$ Department of Oncology, Tongji Hospital, Tongji Medical College, Huazhong University of Science and Technology, Wuhan, China.

Received: 5 October 2021 Accepted: 24 February 2022

Published online: 08 March 2022

\section{References}

1. Huang C, Wang Y, Li X, Ren L, Zhao J, Hu Y, et al. Clinical features of patients infected with 2019 novel coronavirus in Wuhan, China. Lancet (London England). 2020;395(10223):497-506.

2. WHO. Coronavirus disease. (COVID-19) outbreak situation 2021 [Available from: https://www.who.int/publications/m/item/weekly-opera tional-update-on-covid-19-31-may-2021.

3. Ni W, Yang X, Yang D, Bao J, Li R, Xiao Y, et al. Role of angiotensin-converting enzyme 2 (ACE2) in COVID-19. Critical care (London, England). 2020;24(1):422.

4. Fanelli V, Fiorentino M, Cantaluppi V, Gesualdo L, Stallone G, Ronco C, et al. Acute kidney injury in SARS-CoV-2 infected patients. Crit Care (London England). 2020;24(1):155

5. Lamers M, Beumer J, van der Vaart J, Knoops K, Puschhof J, Breugem T, et al. SARS-CoV-2 productively infects human gut enterocytes. Science. 2020;369(6499):50-4.

6. Lindner D, Fitzek A, Bräuninger H, Aleshcheva G, Edler C, Meissner K, et al. Association of Cardiac Infection With SARS-CoV-2 in Confirmed COVID-19 Autopsy Cases. JAMA Cardiol. 2020;5(11):1281-5.

7. Li B, Yang J, Zhao F, Zhi L, Wang X, Liu L, et al. Prevalence and impact of cardiovascular metabolic diseases on COVID-19 in China. Clin Res cardiology: official J German Cardiac Soc. 2020;109(5):531-8.

8. Wang Z, XX. scRNA-seq Profiling of Human Testes Reveals the Presence of the ACE2 Receptor, A Target for SARS-CoV-2 Infection in Spermatogonia, Leydig and Sertoli Cells. Cells. 2020;9(4):920.

9. Kopel J, Perisetti A, Roghani A, Aziz M, Gajendran M, Goyal H. Racial and Gender-Based Differences in COVID-19. Front Public Health. 2020;8:418.

10. Erbay G, Sanli A, Turel H, Yavuz U, Erdogan A, Karabakan M, et al. Shortterm effects of COVID-19 on semen parameters: A multicenter study of 69 cases. Andrology. 2021;9(4):1060-5.

11. Guo T, Sang $c$, Bai $S, M a H, v Y$, Jiang $X$. Semen parameters in men recovered from COVID-19. Asian journal of andrology. 2021;23(5):479-83.

12. Cooper TG, Noonan E, von Eckardstein S, Auger J, Baker HW, Behre HM, et al. World Health Organization reference values for human semen characteristics. Hum Reprod Update. 2010;16(3):231-45.

13. Wang M, Zhu L, Liu C, He H, Wang C, Xing C, et al. A Novel Assisted Oocyte Activation Method Improves Fertilization in Patients With Recurrent Fertilization Failure. Front cell Dev biology. 2021;9:672081.

14. Wang M, Xi Q, Yang Q, Li Z, Yang L, Zhu L, et al. The relationship between a novel evaluation parameter of premature luteinization and IVF outcomes. Reprod Biomed Online. 2021;42(2):323-31.

15. Braga D, Setti A, Figueira R, laconelli A, Borges $E$. The importance of the cleavage stage morphology evaluation for blastocyst transfer in patients with good prognosis. J Assist Reprod Genet. 2014;31(8):1105-10.

16. Gardner D, Schoolcraft W. Culture and transfer of human blastocysts. Curr Opin Obstet Gynecol. 1999;11(3):307-11.

17. Xie J, Ding C, Li J, Wang Y, Guo H, Lu Z, et al. Characteristics of patients with coronavirus disease (COVID-19) confirmed using an IgM-lgG antibody test. J Med Virol. 2020;92(10):2004-10.

18. Xiang F, Wang X, He X, Peng Z, Yang B, Zhang J, et al. Antibody Detection and Dynamic Characteristics in Patients With Coronavirus Disease 2019. Clin Infect diseases: official publication Infect Dis Soc Am. 2020:71(8):1930-4.
19. Du T, Wang Y, Fan Y, Zhang S, Yan Z, Yu W, et al. Fertility and neonatal outcomes of embryos achieving blastulation on Day 7: are they of clinical value? Human reproduction (Oxford. England). 2018;33(6):1038-51.

20. Hoffmann M, Kleine-Weber H, Schroeder S, Krüger N, Herrler T, Erichsen S, et al. SARS-CoV-2 Cell Entry Depends on ACE2 and TMPRSS2 and Is Blocked by a Clinically Proven Protease Inhibitor. Cell. 2020;181(2):271 $-80 . e 8$.

21. Li M, Li L, Zhang Y, Wang X. Expression of the SARS-CoV-2 cell receptor gene ACE2 in a wide variety of human tissues. Infect Dis poverty. 2020;9(1):45.

22. Ruan Y, Hu B, Liu Z, Liu K, Jiang H, Li H, et al. No detection of SARS-CoV-2 from urine, expressed prostatic secretions, and semen in 74 recovered COVID-19 male patients: A perspective and urogenital evaluation. Andrology. 2021;9(1):99-106.

23. Song C, Wang Y, Li W, Hu B, Chen G, Xia P, et al. Absence of 2019 novel coronavirus in semen and testes of COVID-19 patientst. Biol Reprod. 2020;103(1):4-6.

24. Pan F, Xiao X, Guo J, Song Y, Li H, Patel D, et al. No evidence of severe acute respiratory syndrome-coronavirus 2 in semen of males recovering from coronavirus disease 2019. Fertil Steril. 2020;113(6):1135-9.

25. Guo L, Zhao S, Li W, Wang Y, Li L, Jiang S, et al. Absence of SARS-CoV-2 in semen of a COVID-19 patient cohort. Andrology. 2021;9(1):42-7.

26. Holtmann N, Edimiris P, Andree M, Doehmen C, Baston-Buest D, Adams $\mathrm{O}$, et al. Assessment of SARS-CoV-2 in human semen-a cohort study. Fertil Steril. 2020;114(2):233-8.

27. Kayaaslan B, Korukluoglu G, Hasanoglu I, Kalem A, Eser F, Akinci E, et al. Semen Does Not Cause Additional Risk for SARS-CoV-2 Transmission during Sexual Contact. Urol Int. 2020;104:1003-4.

28. Li D, Jin M, Bao P, Zhao W, Zhang S. Clinical Characteristics and Results of Semen Tests Among Men With Coronavirus Disease 2019. JAMA Netw open. 2020;3(5):e208292.

29. Temiz M, Dincer M, Hacibey I, Yazar R, Celik C, Kucuk S, et al. Investigation of SARS-CoV-2 in semen samples and the effects of COVID-19 on male sexual health by using semen analysis and serum male hormone profile: A cross-sectional, pilot study. Andrologia. 2021;53(2):e13912.

30. Gacci M, Coppi M, Baldi E, Sebastianelli A, Zaccaro C, Morselli S, et al. Semen impairment and occurrence of SARS-CoV-2 virus in semen after recovery from COVID-19. Human reproduction (Oxford, England). 2021;36(6):1520-9.

31. Li H, Xiao X, Zhang J, Zafar M, Wu C, Long Y, et al. Impaired spermatogenesis in COVID-19 patients. EClinicalMedicine. 2020;28:100604.

32. Best J, Kuchakulla M, Khodamoradi K, Lima T, Frech F, Achua J, et al. Evaluation of SARS-CoV-2 in Human Semen and Effect on Total Sperm Number: A Prospective Observational Study. The world journal of men's health. 2021;39(3):489-95.

33. Wiersinga W, Rhodes A, Cheng A, Peacock S, Prescott H. Pathophysiology. Transmission, Diagnosis, and Treatment of Coronavirus Disease 2019 (COVID-19): A Review. JAMA. 2020;324(8):782-93.

34. Kariuki S, Selhorst P, Norman J, Cohen K, Rebe K, Williamson C, et al. Detectable HIV-1 in semen in individuals with very low blood viral loads. Virol J. 2020;17(1):29.

35. Zhao S, Zhu W, Xue S, Han D. Testicular defense systems: immune privilege and innate immunity. Cell Mol Immunol. 2014;11(5):428-37.

36. Tan L, Lacko L, Zhou T, Tomoiaga D, Hurtado R, Zhang T, et al. Pre- and peri-implantation Zika virus infection impairs fetal development by targeting trophectoderm cells. Nat Commun. 2019;10(1):4155.

37. Li R, Yin T, Fang F, Li Q, Chen J, Wang Y, et al. Potential risks of SARSCoV-2 infection on reproductive health. Reprod Biomed Online. 2020;41(1):89-95.

38. Wang $M$, Yang $Q$, Ren $X$, Hu J, Li Z, Long R, et al. Investigating the impact of asymptomatic or mild SARS-CoV-2 infection on female fertility and in vitro fertilization outcomes: A retrospective cohort study. EClinicalMedicine. 2021;38:101013.

39. Lucas E. Epigenetic effects on the embryo as a result of periconceptional environment and assisted reproduction technology. Reprod Biomed Online. 2013;27(5):477-85.

40. Wong C, Loewke K, Bossert N, Behr B, De Jonge C, Baer T, et al. Noninvasive imaging of human embryos before embryonic genome activation predicts development to the blastocyst stage. Nat Biotechnol. 2010;28(10):1115-21. 
41. Eckersley-Maslin M, Alda-Catalinas C, Reik W. Dynamics of the epigenetic landscape during the maternal-to-zygotic transition. Nat Rev Mol Cell Biol. 2018;19(7):436-50.

42. Wu J, Xu J, Liu B, Yao G, Wang P, Lin Z, et al. Chromatin analysis in human early development reveals epigenetic transition during ZGA. Nature. 2018;557(7704):256-60.

43. Li L, Li L, Li Q, Liu X, Ma X, Yong J, et al. Dissecting the epigenomic dynamics of human fetal germ cell development at single-cell resolution. Cell Res. 2021;31(4):463-77.

44. Xia W, Xu J, Yu G, Yao G, Xu K, Ma X, et al. Resetting histone modifications during human parental-to-zygotic transition. Sci (New York NY). 2019;365(6451):353-60.

\section{Publisher's Note}

Springer Nature remains neutral with regard to jurisdictional claims in published maps and institutional affiliations.

- fast, convenient online submission

- thorough peer review by experienced researchers in your field

- rapid publication on acceptance

- support for research data, including large and complex data types

- gold Open Access which fosters wider collaboration and increased citations

- maximum visibility for your research: over $100 \mathrm{M}$ website views per year

At $\mathrm{BMC}$, research is always in progress.

Learn more biomedcentral.com/submissions 